\title{
Stadtforschung, der schwierige Weg von der Erkenntnis zur Umsetzung
}

\author{
Stefan Emeis (IMK-IFU) + Joachim Fallmann (IMK-TRO) \\ Karlsruher Institut für Technologie, Garmisch-Partenkirchen + Karlsruhe \\ stefan.emeis@kit.edu
}


Ausgangslage

Atmosphärische Stadtforschung seit über 200 Jahren (Howard 1818-20).

Umfasst u.a.:

- städtische Luftqualität

- Strahlungsklima

- Windverhältnisse

- städtische Wärmeinsel

Erkenntnisse aus der Stadtklimaforschung fanden bisher nur begrenzt Eingang in die Stadtplanung (z.B. Eliasson 2000; Mills et al. 2010; Parasee et al. 2019).

Mindestens vier Bereiche müssen reagieren:

- Baumaterialien und Gebäudegestaltung

- Grün und Blau in der Stadt

- Stadtplanung (Siedlungsstruktur, Verkehr, ...)

- Einbindung der Städte in regionale/überregionale Infrastrukturen. 
Jahrtausendealte Ideen prägen unsere Städte. Es dominieren:

- wirtschaftliche Interessen,

- technische Funktionalitäten, Normen, Gesetze

- Wohnbedarf

- Verkehr,

- Prestige und Ästhetik

aber bisher nicht ausreichend:

- Nachhaltigkeit

- gesundes Lokalklima

- Klimaresilienz

Klimaforschung kann viele Ideen beisteuern, damit unsere Bauten und Städte nachhaltiger und klimaresilienter werden,

\section{aber wie bringt man sie in die Umsetzung?}




\section{Hinweis:}

Der Erstautor (S.E.) betrachtet die Fragestellung aus drei Blickrichtungen:

- aus der Sicht eines Atmosphärenwissenschaftlers

- aus der Sicht eines Obmannes eines Richtlinienausschusses der KRdL

- aus der Sicht eines Stadtrates und eines Kreisrates.

Der Zweitautor (J.F.) betrachtet

- aus der Sicht eines Geographen 


\section{aus der Sicht eines Atmosphärenwissenschaftlers:}

12 Herausforderungen und Notwendigkeiten für urbane Gebiete

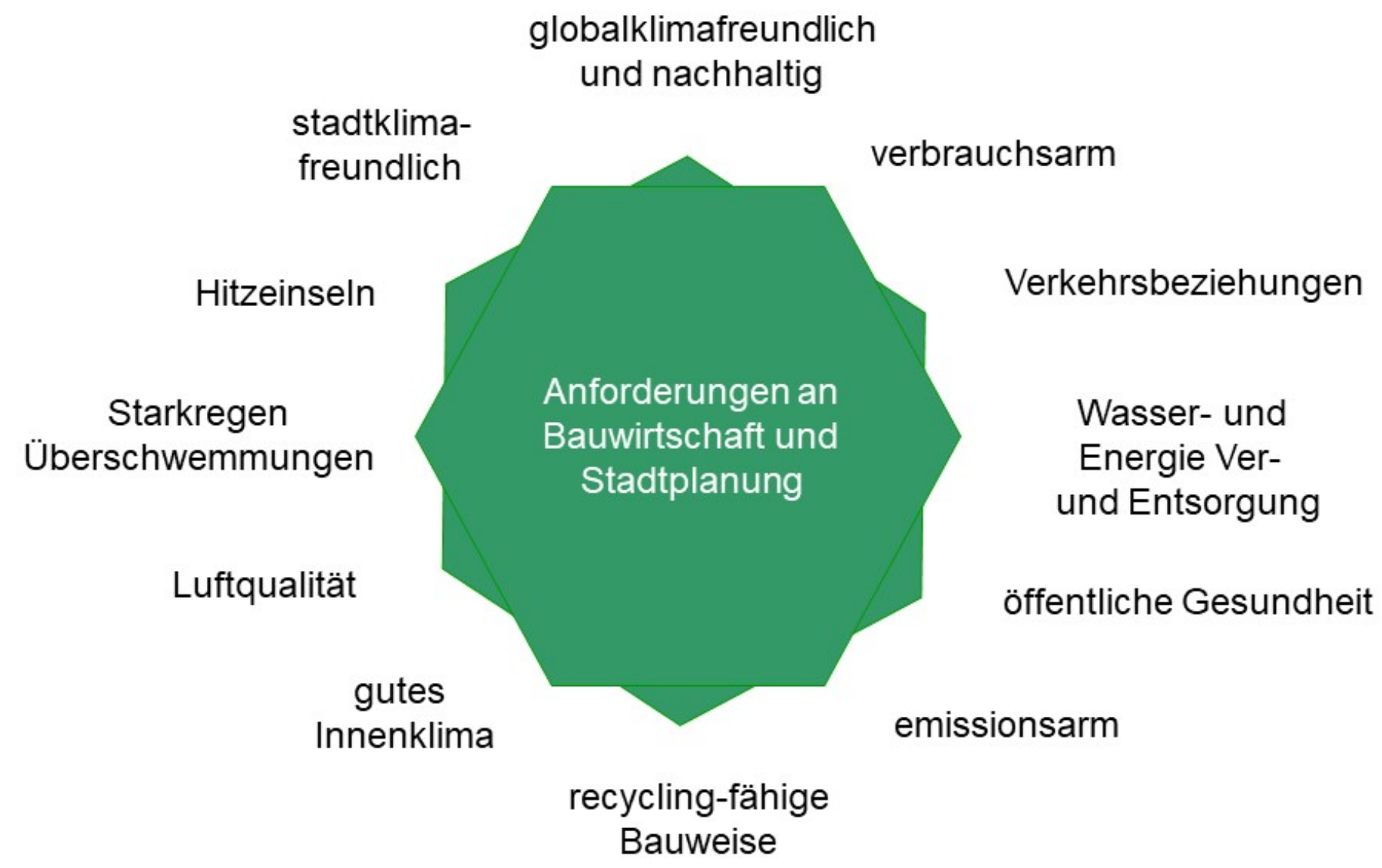




\section{aus der Sicht der Planer:}

\section{7}

Herausforderungen und Notwendigkeiten für urbane Gebiete

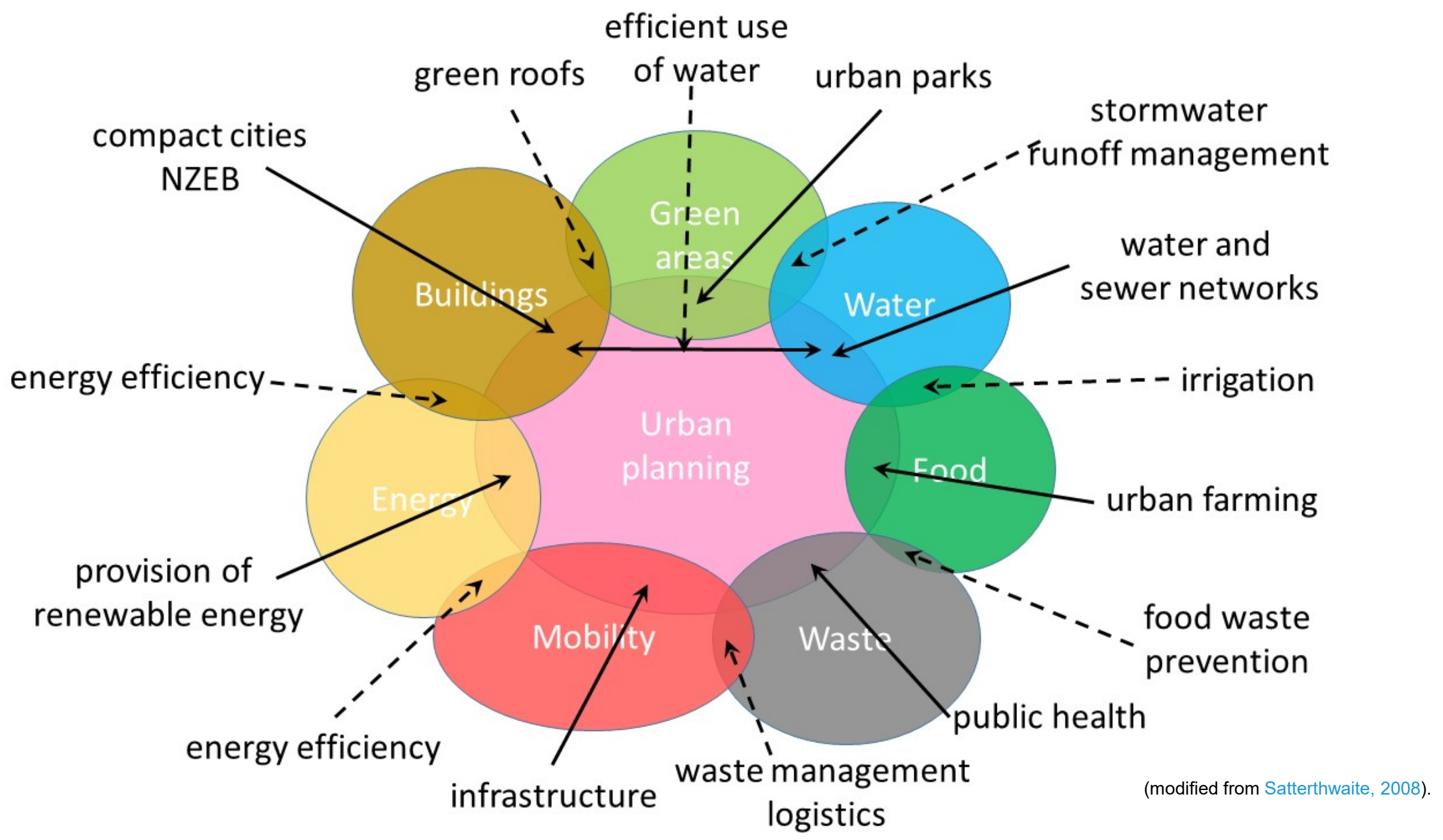

Fallmann, J., S. Emeis, 2020: How to Bring Urban and Global Climate Studies together with Urban Planning and Architecture? Devel. Built Environ., 4, 100023 


\section{Beides zusammen:}

10

Herausforderungen und Notwendigkeiten für urbane Gebiete

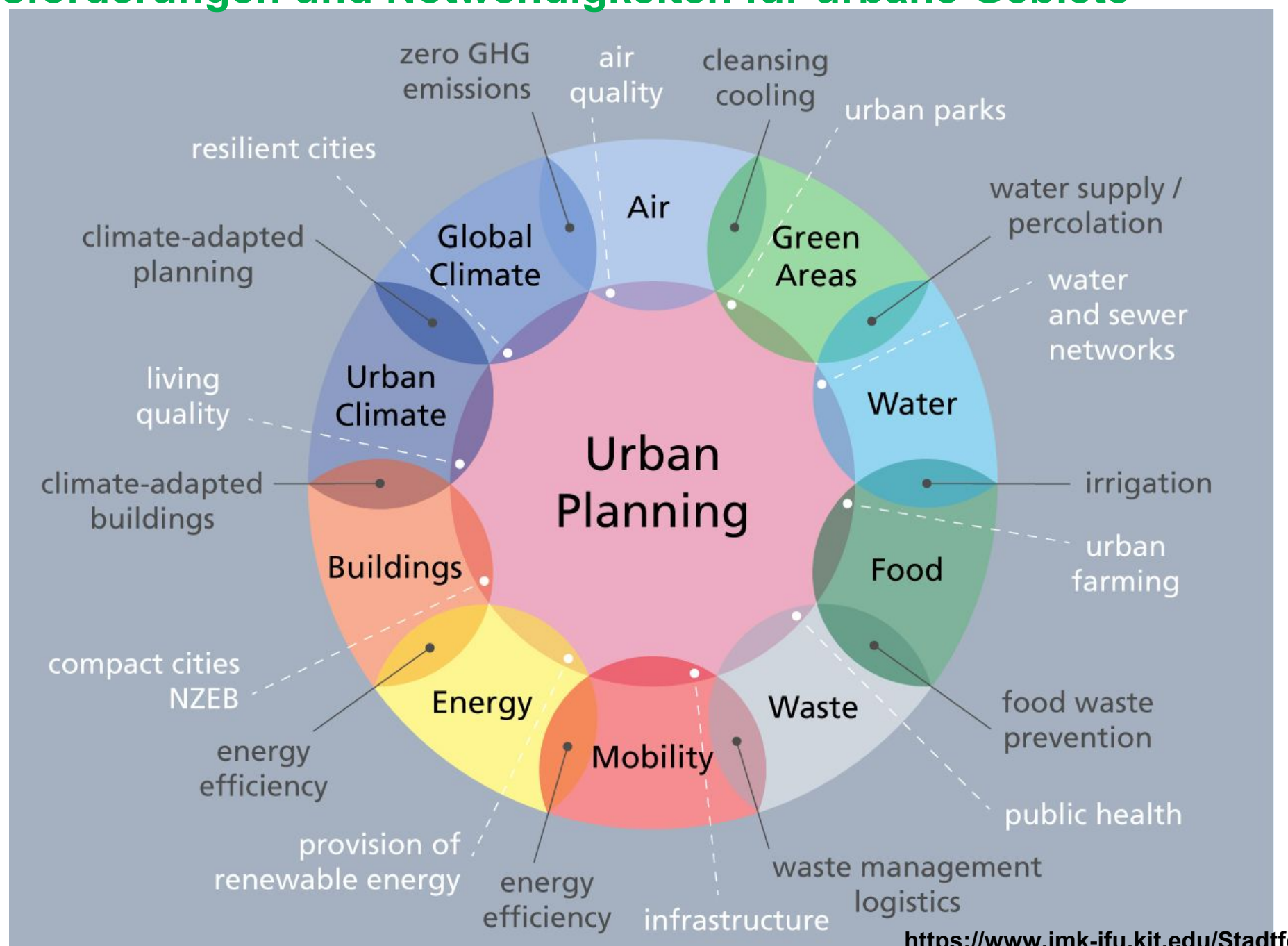

https://www.imk-ifu.kit.edu/Stadtforschung.php 


\section{Beides zusammen:}

eigentlich eine überschaubare Aufgabe (siehe Fallmann und Emeis, 2020)

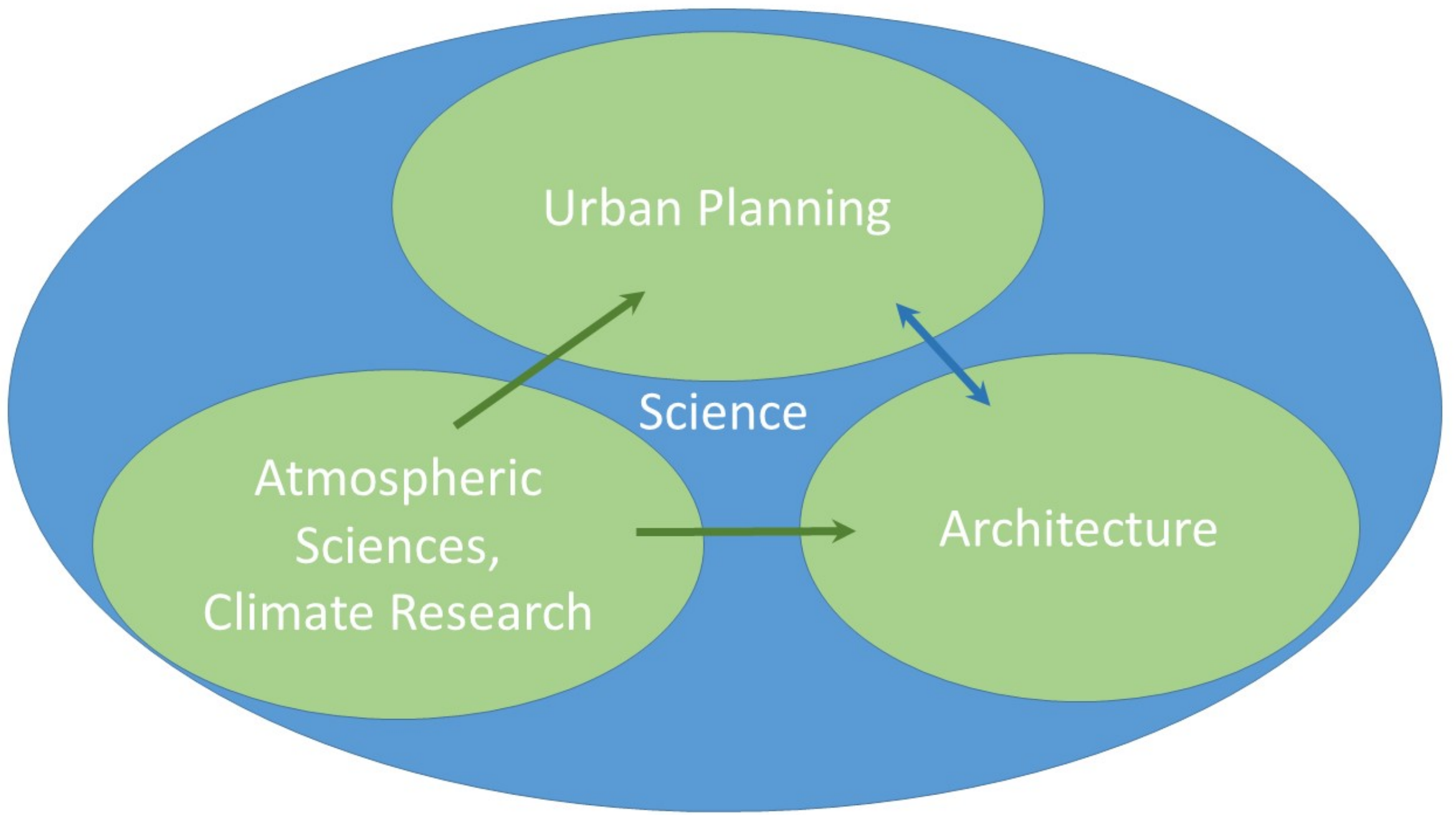




\section{Zusammenfassung (Grafik: Joachim Fallmann)}

\section{Ergebnisse des Reviews - Ranking aus meteorologischer Perspektive}

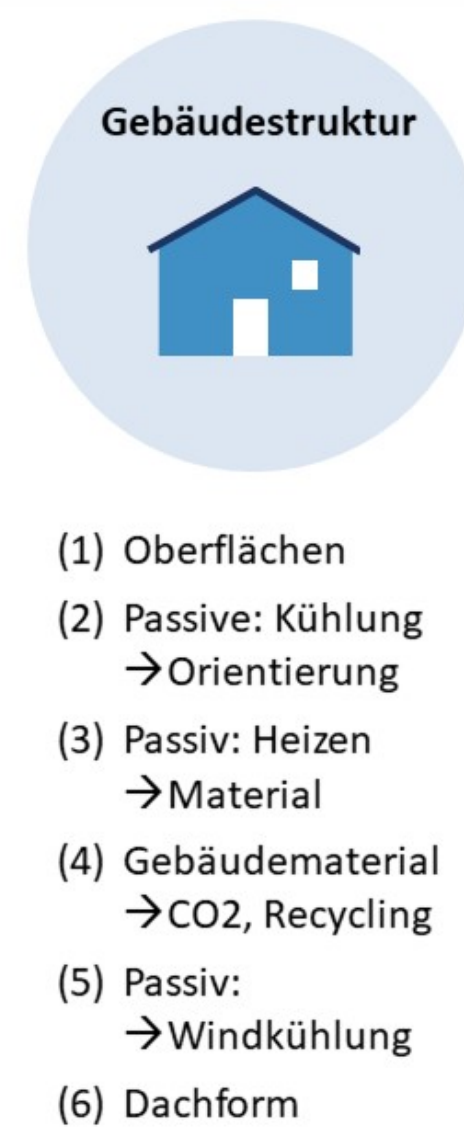

Fallmann, J. and Emeis, S., 2020. How to Bring Urban and Global
Climate Studies together with Urban Planning and Architecture?. Developments in the Built Environment, p.100023.

(1) Oberflächen

(2) Passive: Kühlung $\rightarrow$ Orientierung

(1) Grün am Gebäude

(2) Stadtgrün

(3) Wasserflächen

(4) Urban Gardening

Grün und blau

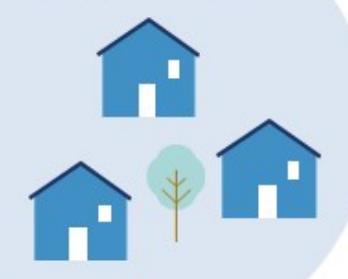

(1) Energieversorgung

(2) Frischluft

(3) Verdichtung

(4) Öffentlicher Nahverkehr

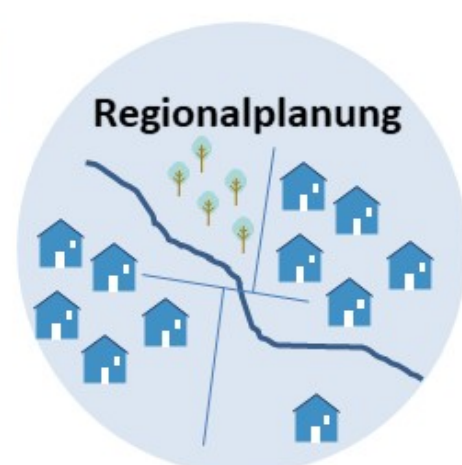

(1) Verkehr

(2) Klimatische Extrem-Events 


\section{Fassadenbegrünung (Beispiel 'Bosco Verticale’, Mailand)}

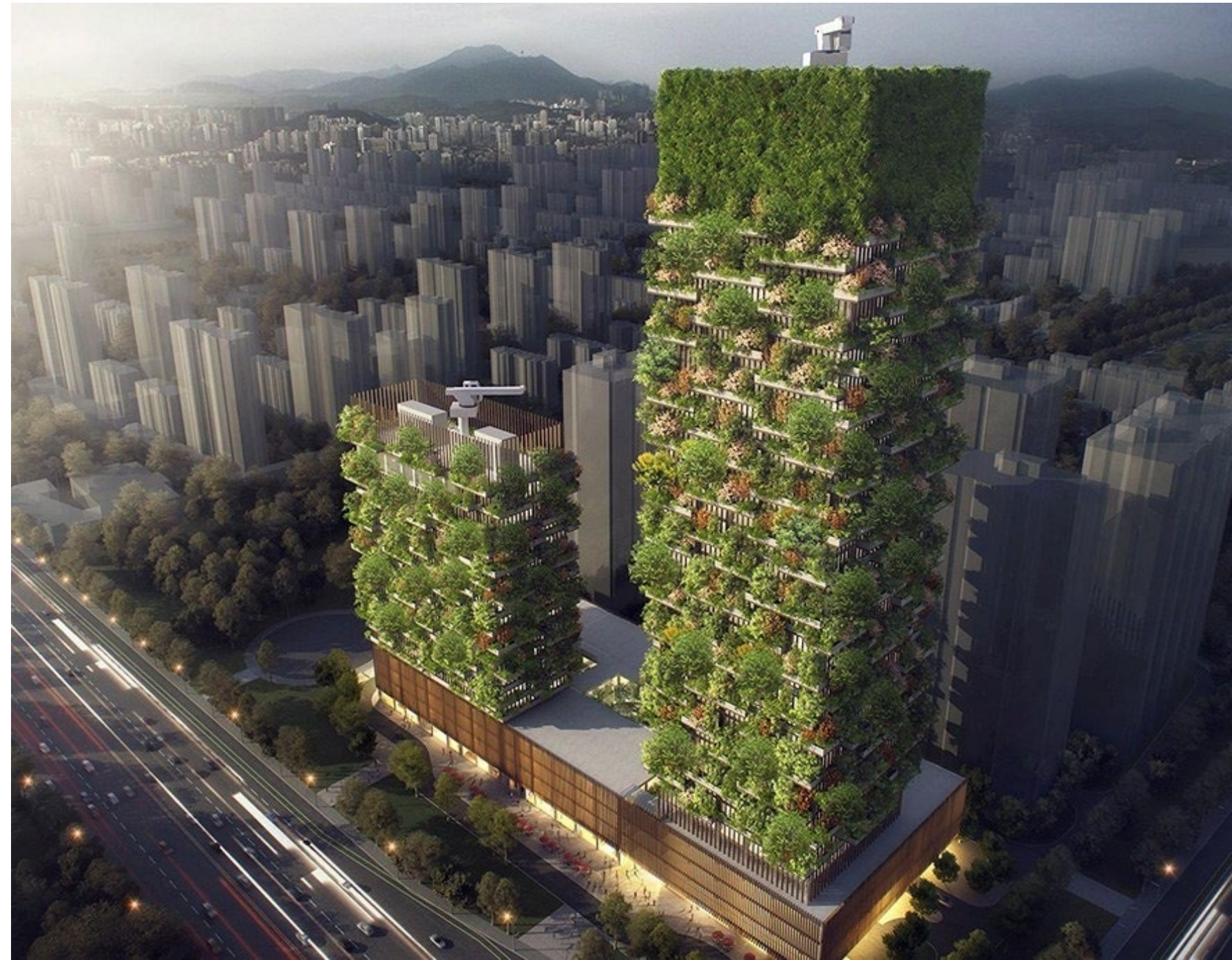

Source: www.stefanoboeriarchitetti.net 


\section{Wüstenstädte ... - Belüftung durch Windtürme (badgir, )}

$$
\text { بادكير }
$$

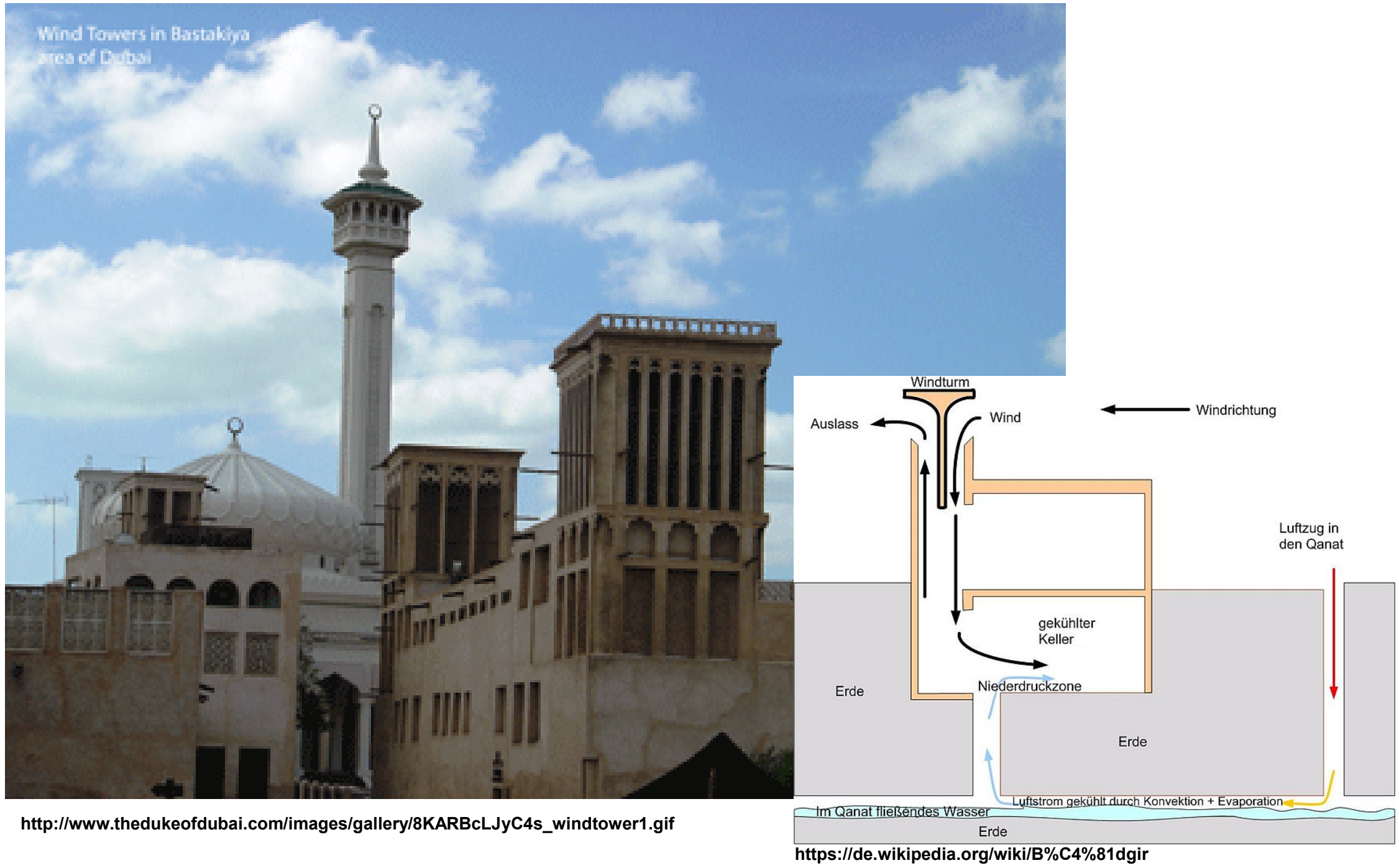


Weitere Punkte

die interdisziplinär entwickelt werden sollten:

\section{Stadtplanung}

Energieversorgung

Frischluftschneisen

Reduktion der Zersiedelung

Abkehr von strikter funktionaler Trennung (von „Athen“ zu „Leipzig“)

Ausbau und Privilegierung des ÖPNV
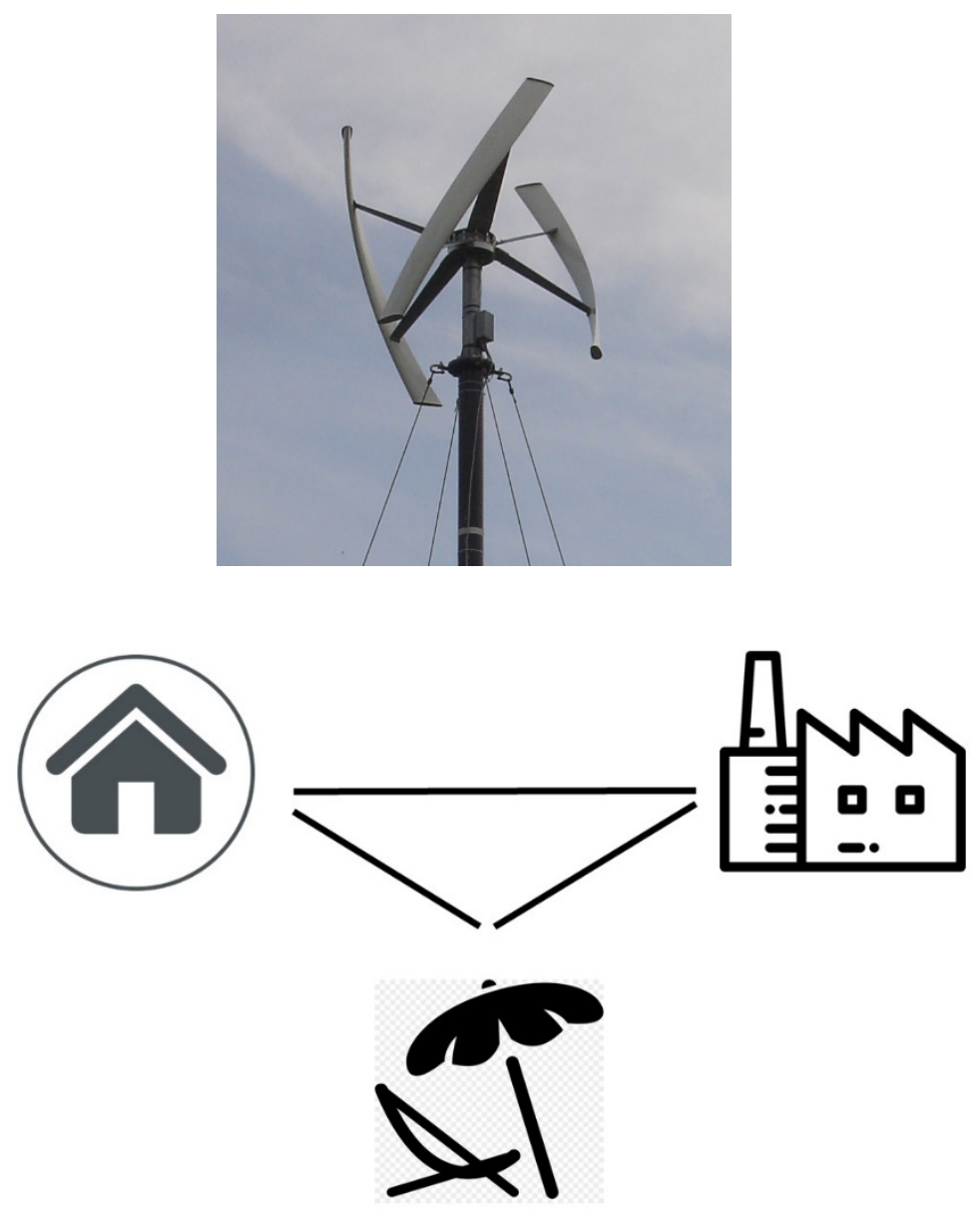


\section{Aber:}

Städte und Länder haben ihre interne Struktur ...

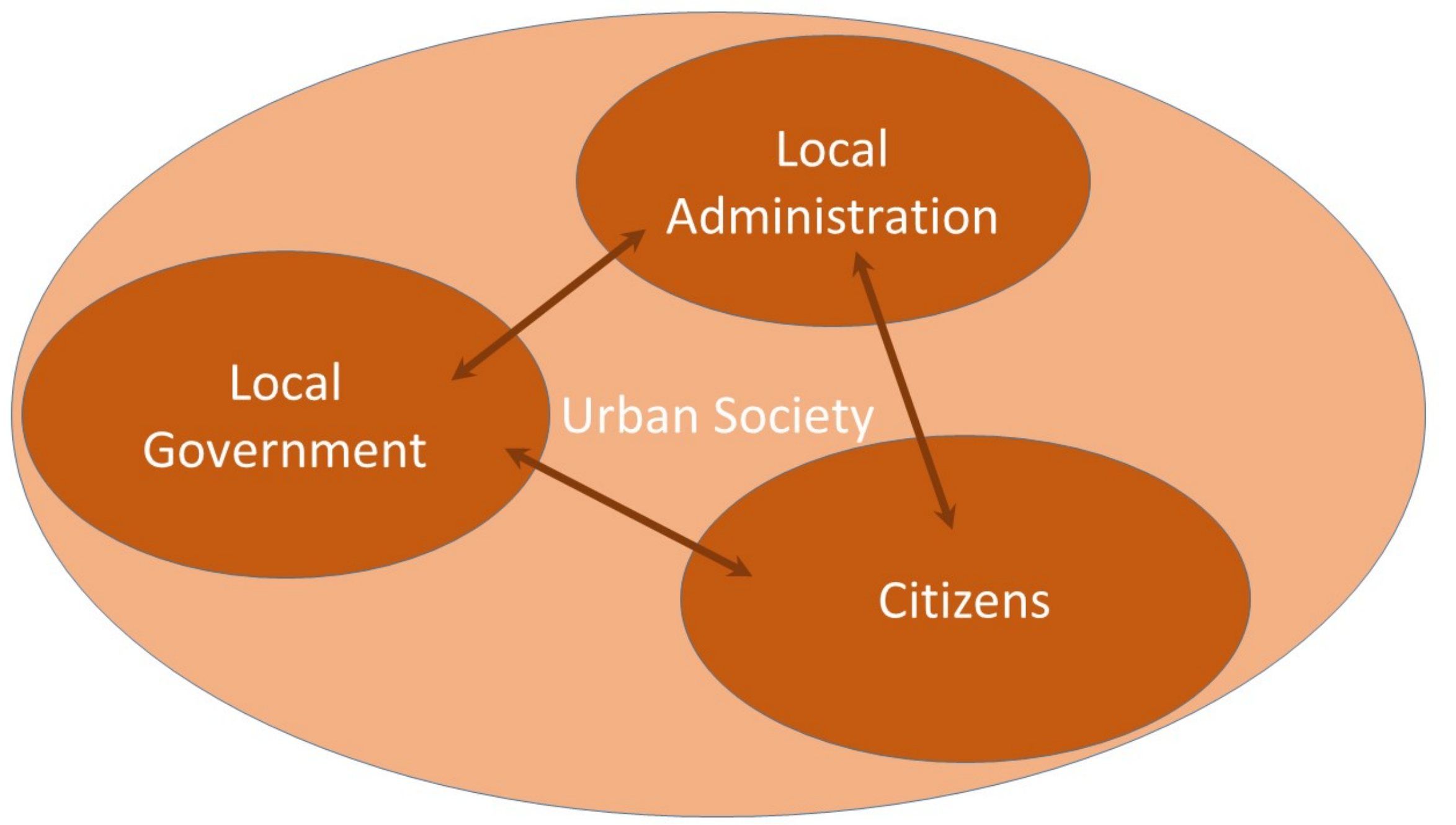




\section{Aber:}

und es gibt viele übergeordnete Strukturen und Regelwerke ...

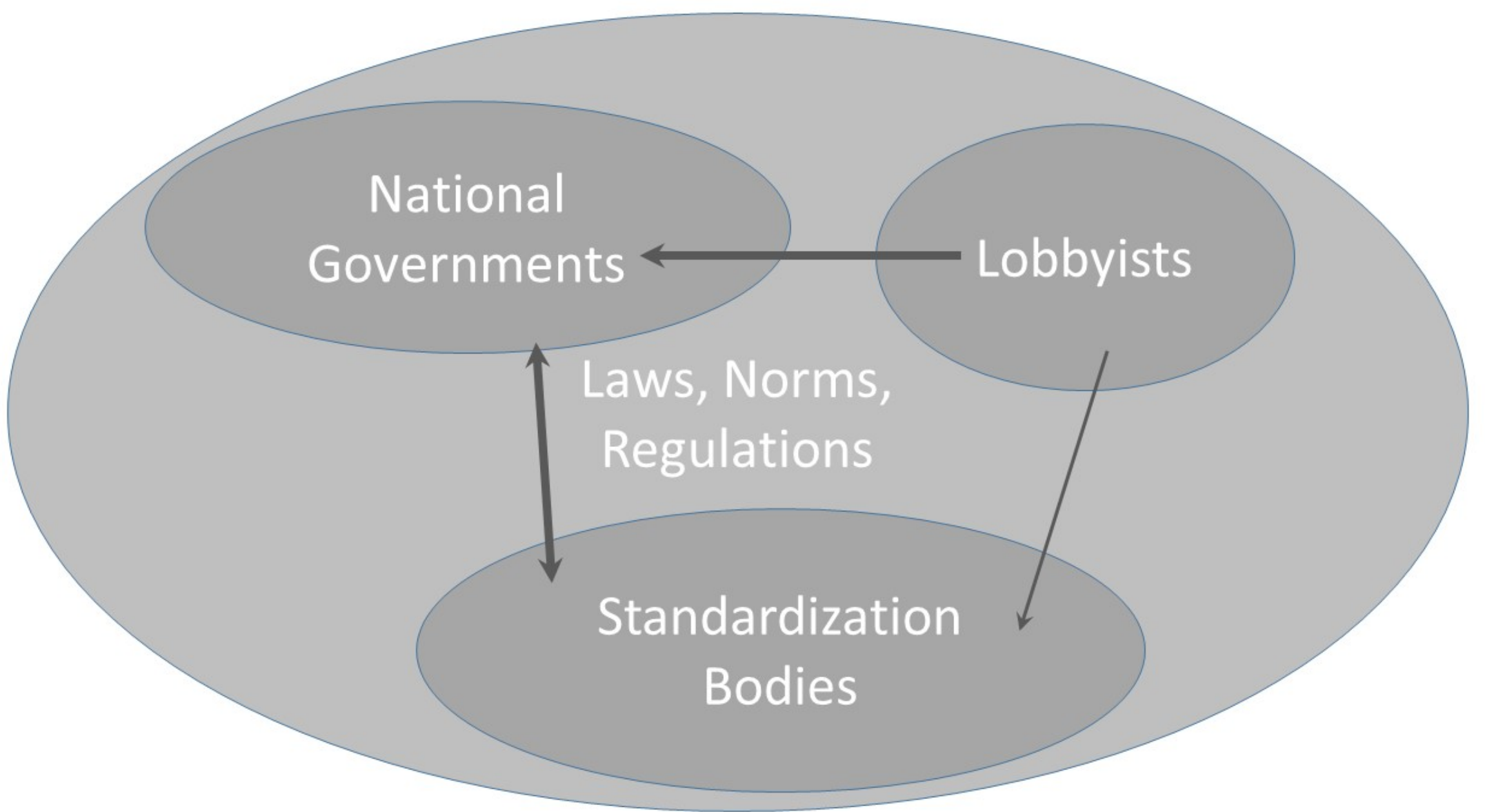


... wobei der Einfluss der Lobbyisten gar nicht immer so negativ sein muss ...

Cartoon von Charles W. Saalburg vom 14. März 1891 auf der Titelseite des Wasp (San Francisco)

Lobbyisten bedrängen einen Stadtverordneten

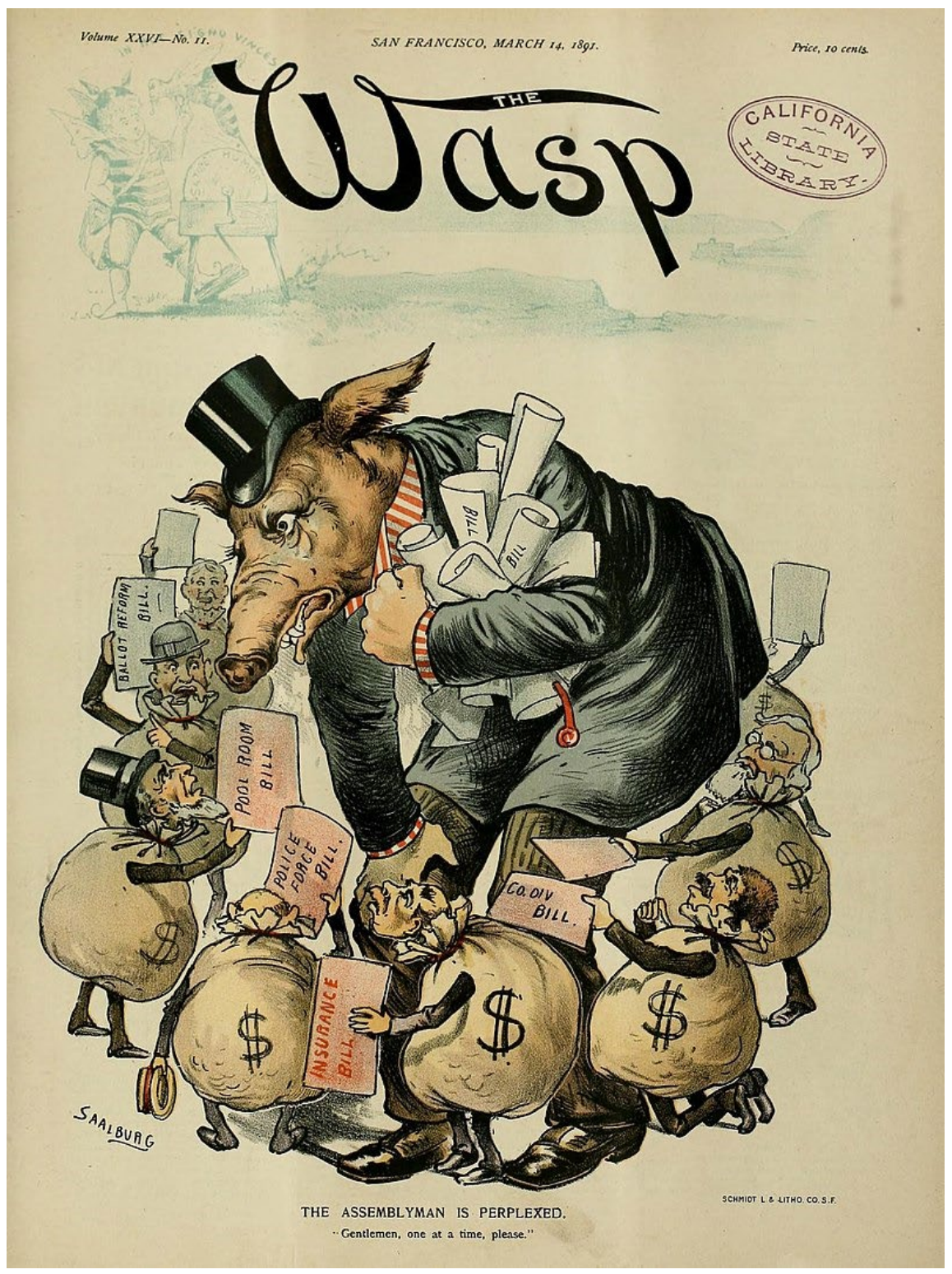




\section{Konsequenz:}

\section{ein kompliziertes Dreieck zwischen Wissenschaft, Gesellschaft und Normen}

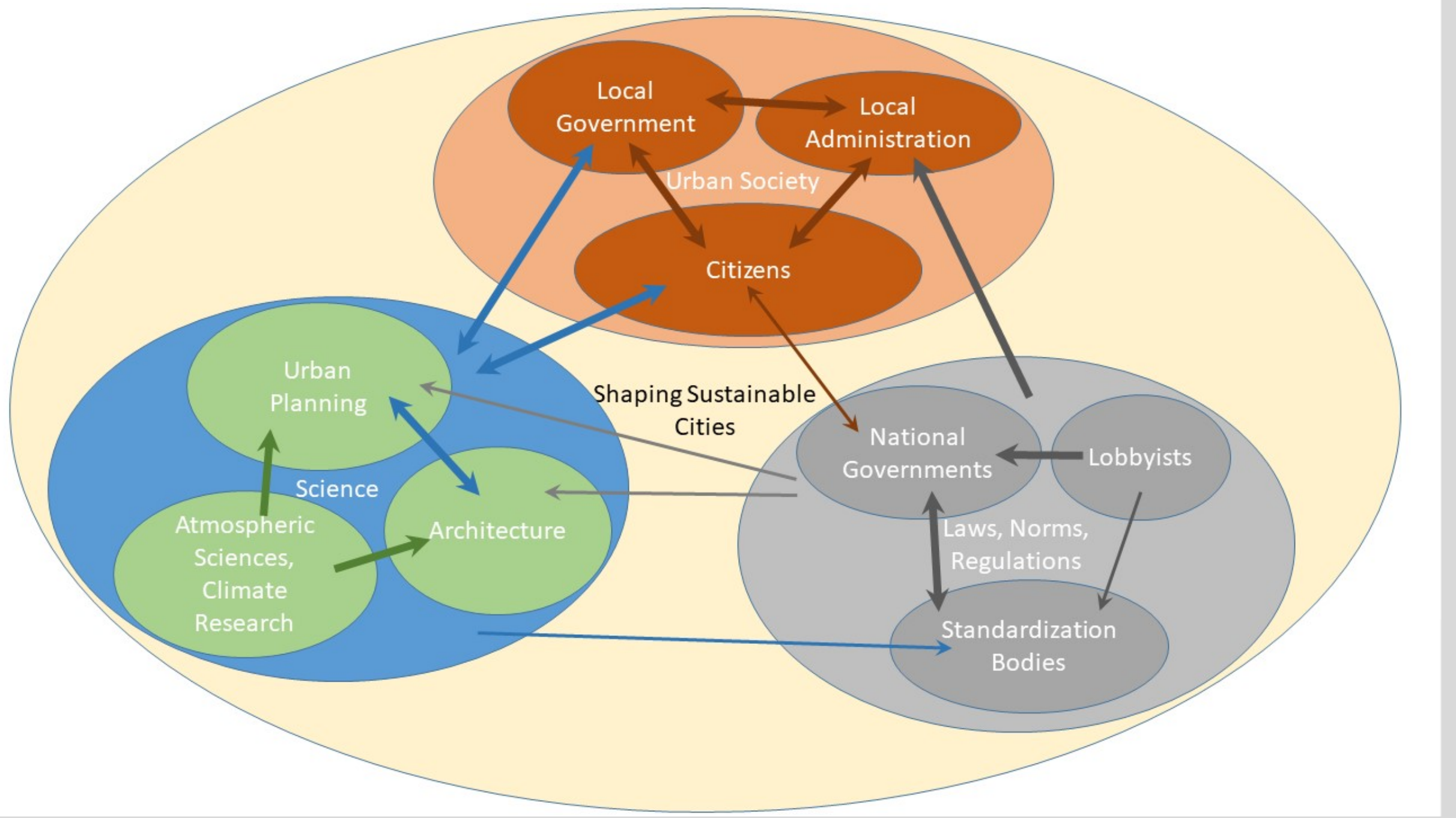




\section{Solange Gebäude wie der „Fryscraper“ in London gebaut werden können ...}

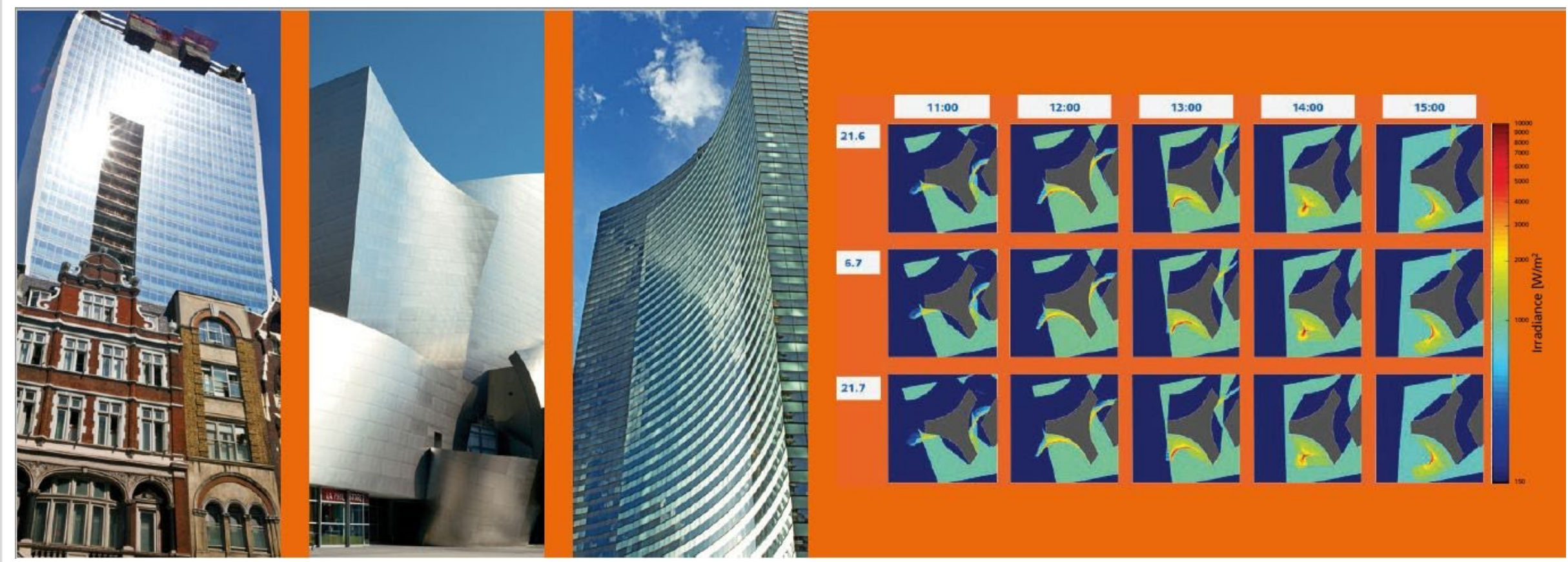

de Boer, J., Fang, Y., Wössnner, S., 2017: IBP-Mitteilungen 550. Fraunhofer Institut für Bauphysik

... werden die falschen Prioritäten gesetzt 


\section{Ein kleines Beispiel aus Deutschland: Weiße Dächer}

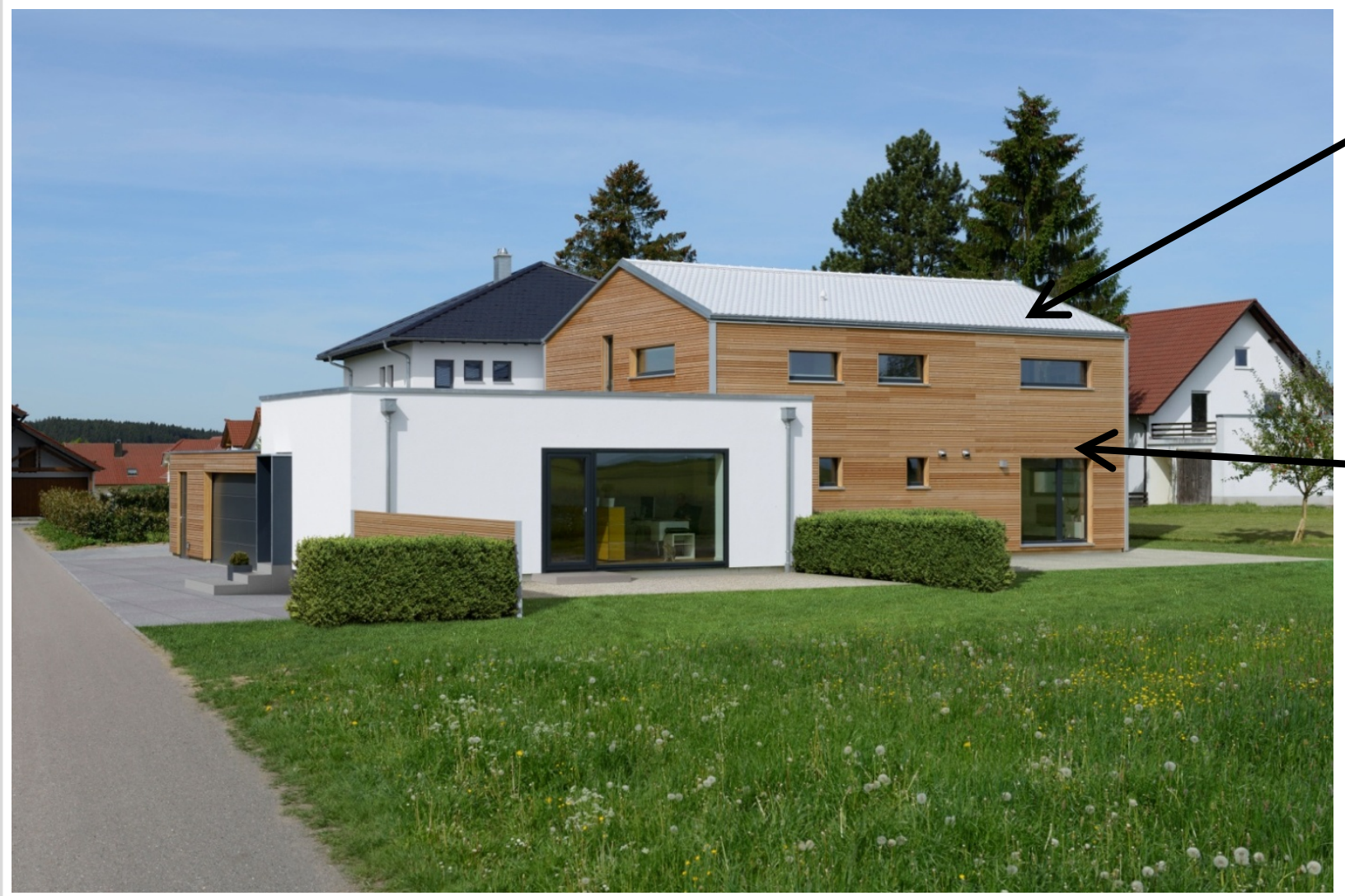

Quelle: Baufritz, http://www.baufritz.com/lu/architektenhaus-mit-weissem-klimaschutz-dach

aber noch unüblich und weiße Dächer durch Bauordnungen oft nicht zugelassen 


\section{Notwendige Ansätze 1:}

\section{Wissenschaft:}

interdisziplinäre Zusammenarbeit

transdisziplinäre Lösungen

ganzheitliche (holistische) Betrachtungsweisen

Analysen von vollständigen Stoffkreisläufen

Lebenszyklusmodelle

Erdsystemmodelle 
Nicht-lineare Wechselwirkungen zwischen nicht-koordinierten Maßnahmen führen zu Problemen

z.B. verringerte Wärmeinsel vs. Durchmischung $\underset{x}{*}$

daher: Abschaffung aller Verbrennungsvorgänge in Städten notwendig
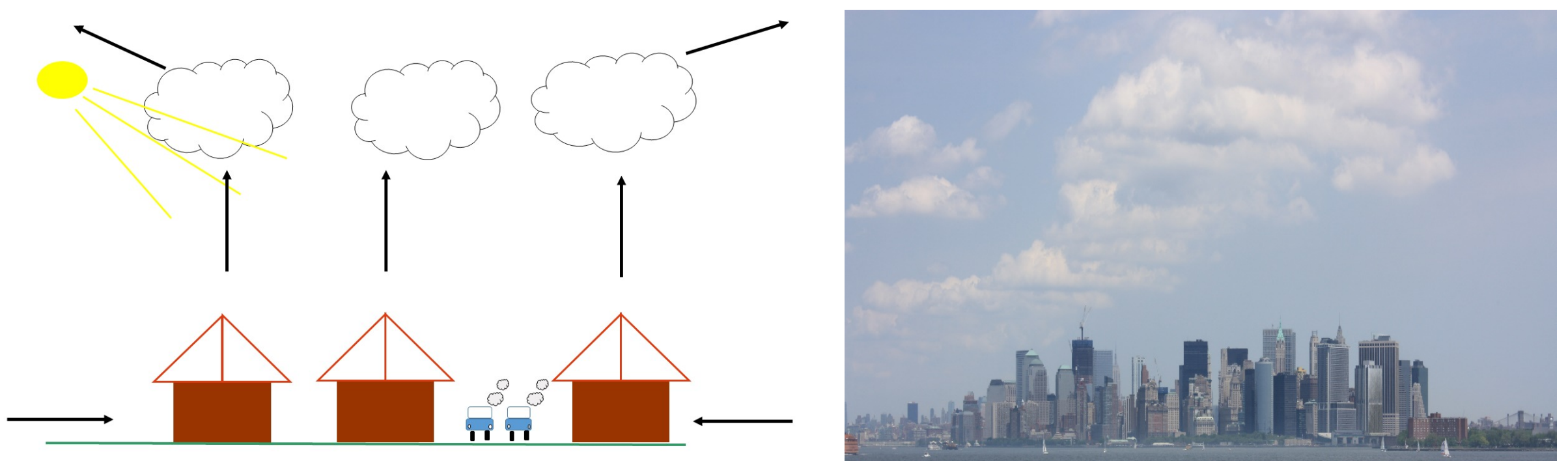

Fallmann, J., R. Forkel, S. Emeis, 2016: Secondary effects of urban heat island mitigation measures on air quality. Atmos. Environ., 125, $199-211$. 



\section{Notwendige Ansätze 2:}

Gesellschaft:

(lokale) Politikberatung

Information großer Teile der Bevölkerung (Abwehr von „fake news“)

Real-Labore für „Best Practice“-Beispiele

Regulatorische Ebene:

Beratung von Politik und "Stakeholdern“" Flexibilisierung von Verordnungen (weiße Dächer!) Unterstützung (möglicherweise Beschleunigung) von Normungsprozessen [arbeiten Sie an der Richtlinienerstellung im z.B. im KRdL des VDI und DIN mit] 


\section{Schlussfolgerung}

Gute Wissenschaft ist eine notwendige Voraussetzung für eine nachhaltige Stadtplanung.

Aber nicht hinreichend.

Die gesellschaftliche und die regulatorische Ebene müssen mitziehen.

Das kann die Wissenschaft alleine nicht leisten (trotz aller zuvor genannten Ansätze).

Sie kann nur die Grundlagen und Fakten dafür liefern. 
Eliasson, I. 2000. The use of climate knowledge in urban planning. Landscape and Urban Planning 48, 1-2, 31-44.

Mills, G; H. Cleugh; R. Emmanuel; W. Endlicher; E. Erell; G. McGranahan; E. Ng; A. Nickson; J. Rosenthal; K. Steemer. 2010. Climate Information for Improved Planning and Management of Mega Cities (Needs Perspective). Procedia Environmental Sciences 1, 228246.

Parsaee, M; M. M. Joybari; P. A. Mirzaei; F. Haghighat. 2019. Urban heat island, urban climate maps and urban development policies and action plans. Environmental Technology \& Innovation 14, 100341.

Alle drei Arbeiten zeigen, dass die Erkenntnisse der Klimaforschung bisher zu wenig Eingang in die Stadtplanung und die Architektur gefunden haben. 

